\title{
Short-wavelength asymptotics of time correlation functions
}

\author{
V.V.Ignatyuk \\ Institute for Condensed Matter Physics \\ of the National Academy of Sciences of Ukraine, \\ 1 Svientsitskii Str., 79011 Lviv, Ukraine
}

Received August 1, 2000

\begin{abstract}
Short-wavelength asymptotics of time correlation functions (TCF) of simple classical fluids is considered in the framework of short time expansion. The explicit expressions for zeroth time moments up to the 8-th order of cumulant are presented in the framework of additive approach. It is shown that unlike the dynamic structure factor which is found to have Gaussian form in short-wavelength limit, nongaussian corrections are present in TCF "density-energy" and "energy-energy". The comparison with generalized collective modes approach as well as data for quantum liquids is discussed.
\end{abstract}

Key words: time correlation functions, short-wavelength limit, generalized collective modes, cumulant expansion

PACS: 05.70.Ln, 51.10.+y, 61.20.Lc, 61.20.Ne

\section{Introduction}

During the last years the investigations of dynamic properties of liquids (simple [1], quantum [2], polar [3], multicomponent [4]) have shown great interest both among theoretical and experimental physicists. First of all they deal with the improvement of scattering techniques in conjunction with the possibility of a more complex computer simulation of the dynamic processes in the systems mentioned above. At the same time, there arises a problem of creating the computer-adapted theories, which could allow one to carry out investigations of the dynamics of liquids taking the microscopic variables with different space and time scales as a basic set. In the case of liquids, such a problem is complicated by the absence of a small parameter in density (or interaction) and the subject of the research should be time correlation functions (TCF) themselves, where one has to take into account all collective effects in fluids.

As a rule, the dynamic structure factor $S(\omega, k)$, directly related to TCF "densitydensity", is one of the experimentally observable values by neutron scattering. For ionic liquids one can study TCF "charge-charge" by light scattering on inhomo- 
geneities of electric field [5]. Light scattering experiments allow one to investigate the dynamics of liquids in the domain of small values of wave vector $k$ and frequency $\omega$, that is in hydrodynamic region. In this domain the dynamic structure factor $S(k, \omega)$ could be expressed via some thermodynamic functions (such as specific heats, compressibility) and transport coefficients (bulk and shear viscosities, thermal conductivity) by the mean of Landau-Plachek relation [6]. When going from hydrodynamic region one has to perform generalization of this formula by considering $k$-dependent thermodynamic functions and $(k, \omega)$-dependent transport coefficients. In such a case, the first of them are expressed through static correlation functions $(\mathrm{SCF})$, while transport coefficients - via memory kernels, that is, TCF related to corresponding dissipative fluxes. Such an approach forms the basis of generalized collective modes conception [7] and demonstrated its efficiency through various investigations in the liquids $[1-4]$.

On the other hand, one can formally expand TCFs in the series of time $t$ and estimate the contribution of every order of the moments [8]. Such a procedure turned out to be the most efficient in the domain of large $k \geqslant 15 \AA^{-1}$, when the interaction of a particular molecule with its surrounding could be neglected. It is possible when the relation $\hbar k \gg F \tau$ is fulfilled, where $F$ denotes the force acting on the molecule, $\tau$ means an effective scattering time and $\hbar$ means Planck constant. From this point of view, it corresponds to incoherent scattering [9]. When k increases, characteristics of dynamic structure factor (line width, amplitudes) cease to oscillate and approach their asymptotical values. Thus, in incoherent scattering it is possible to study oneparticle effects. In quantum liquids, short-wavelength scattering is applied to study the deviation of one-particle distribution function from Maxwellian form [10] or to determine the Bose condensate fraction in superfluid helium [9].

In this paper, we study short-wavelength dynamics of a simple liquid. It will be shown that even in the domain of large $k$, TCF "density-energy" and "energyenergy", unlike dynamic structure factor, could not be written down in the Gaussian form. It should be stressed that short-wavelength asymptotics of these TCFs have not almost been investigated because contrary to dynamic structure factor, TCFs "density-energy" and "energy-energy" are not experimentally observed and, consequently, don't present "independent" interest.

In the next section we investigate TCFs in the domain of large $k$ using short time expansion up to the 8-th order cumulant. We show that the so-called final-state effects $[8-10]$ that correspond to the interaction of the particles with its neighbourhood are negligible in the large $k$ limit. For a dynamic structure factor, this directly results in its Gaussian form, for the other TCFs the only relevant corrections are related to the first and to the second moments of interaction potential. In the additive approach [8] it is possible to obtain the explicit expressions for zero time moments of TCFs "density-energy" and "energy-energy" well convergent almost in all cases. In the last section there is a brief discussion of the obtained results in their connection with the generalized collective modes approach $[7,11]$. 


\section{Short time expansion of TCF}

The subject of our interest are TCF $\Phi_{A B}(k, t)$ constructed on the dynamic variables $\hat{A}(\boldsymbol{k}), \hat{B}(\boldsymbol{k})$ and defined as follows ${ }^{1}$ :

$$
\Phi_{A B}(k, t)=\langle\Delta \hat{A}(\boldsymbol{k}) \exp \{-\mathrm{i} \hat{L} t\} \Delta \hat{B}(-\boldsymbol{k})\rangle ; \quad \Delta \hat{A}(\boldsymbol{k})=\hat{A}(\boldsymbol{k})-\langle\hat{A}(\boldsymbol{k})\rangle,
$$

where i $\hat{L}$ means Liouville operator and averaging is being performed over the equilibrium distribution. For quantum systems, there is a corresponding definition of TCF [13]:

$$
\Phi_{A B}(k, t)=\int_{0}^{1}\langle\Delta \hat{A}(\boldsymbol{k}, t+\mathrm{i} \hbar \beta \tau) \Delta \hat{B}(-\boldsymbol{k})\rangle \mathrm{d} \tau
$$

where $\beta=1 / T$ means inverse temperature (we put Boltzmann constant to be equal to unity) while time dependance of the operators is introduced through Heisenberg representation with Hamiltonian $\hat{H} ; \mathrm{i} \hat{L} \hat{A}(\boldsymbol{k}) \equiv \dot{\hat{A}}(\boldsymbol{k})=\mathrm{i} / \hbar[\hat{H}, \hat{A}(\boldsymbol{k})]$.

Fourier transform of TCF "density-density"

$$
S(k, \omega)=\frac{1}{2 \pi} \int_{-\infty}^{\infty} \exp (-\mathrm{i} \omega t) \Phi_{n n}(k, t) \mathrm{d} t
$$

is directly related to the dynamic structure factor (in quantum case, the Fourier transform of TCF $\Phi_{n n}(k, t)$ defined according to (2) determines symmetrized dynamic structure factor $S_{\mathrm{sym}}(k, \omega)$ [13], which by means of a detailed balance principle could be related to the experimentally observed nonsymmetrized dynamic structure factor).

Let us choose the Fourier transforms of hydrodynamic variables as a basic set: number density

$$
\hat{n}(\boldsymbol{k})=\frac{1}{\sqrt{N}} \sum_{i=1}^{N} \exp \left(\mathrm{i}_{\boldsymbol{k}} \boldsymbol{r}_{i}\right)
$$

longitudinal component (along the wave vector) of momentum density

$$
\hat{j}(\boldsymbol{k})=\frac{1}{\sqrt{N}} \sum_{i=1}^{N} \frac{\boldsymbol{k} \boldsymbol{p}_{i}}{k} \exp \left(\mathrm{i} \boldsymbol{k} \boldsymbol{r}_{i}\right)
$$

and total energy density

$$
\hat{\varepsilon}(\boldsymbol{k})=\frac{1}{\sqrt{N}} \sum_{i=1}^{N}\left(\frac{\boldsymbol{p}_{i}^{2}}{2 m}+\sum_{j \neq i=1}^{N} U\left(\left|\boldsymbol{r}_{i}-\boldsymbol{r}_{j}\right|\right)\right) \exp \left(\mathrm{i} \boldsymbol{k} \boldsymbol{r}_{i}\right),
$$

\footnotetext{
${ }^{1}$ We will use the abbreviation TCF for Fourier transforms of the corresponding time correlation functions as well.
} 
where $\boldsymbol{r}_{i}$ and $\boldsymbol{p}_{i}$ denote the position and the momentum of the i-th particle, $m$ means the mass of the particle, $N$ means the total particle number and $U\left(\left|\boldsymbol{r}_{i}-\boldsymbol{r}_{j}\right|\right)$ denotes the total interaction potential of the system.

TCF (1) could be formally expanded in series of $t$ and then presented as a cumulant exponent $[8,9,12]$

$$
\Phi_{A B}(k, t)=M_{0}(k) \exp \left\{\sum_{s=1}^{\infty}(-1)^{s} \frac{t^{2 s}}{(2 s) !} K_{2 s}(k)\right\},
$$

where $M_{0}(k)=\Phi_{A B}(k, t=0)$, and cumulants $K_{s}(k)$ are related with the corresponding moments

$$
M_{2 s}(k)=\frac{\left\langle(\mathrm{i} \hat{L})^{s} \hat{A}(\boldsymbol{k})(\mathrm{i} \hat{L})^{s} \hat{B}(-\boldsymbol{k})\right\rangle}{M_{0}(k)}
$$

in the well-known manner [14]:

$$
\begin{gathered}
K_{2}=M_{2} ; \quad K_{4}=M_{4}-3 M_{2}^{2} ; \quad K_{6}=M_{6}-15 M_{4} M_{2}+30 M_{2}^{3} ; \\
K_{8}=M_{8}-28 M_{2} M_{6}-35 M_{4}^{2}+420 M_{4} M_{2}^{2}-630 M_{2}^{4} .
\end{gathered}
$$

Obviously, it is difficult to explore the expression (7) in calculation of time and frequency moments of TCFs. Frequently, additive approximation for short time expansion of TCFs is used [8-10]:

$$
\Phi_{A B}(k, t)=M_{0}(k) \exp \left[-1 / 2 K_{2}(k) t^{2}\right]\left\{1+\sum_{s=2}^{\infty}(-1)^{s} \frac{t^{2 s}}{(2 s) !} K_{2 s}(k)\right\} .
$$

Now, all the necessary integration could be easily performed: one can obtain analytic expressions both for time and frequency moments of (10). The subject of our interest are zeroth time moments of TCFs because exactly these functions along with SCFs define the elements of a dynamic matrix in the generalized collective modes approach [7]. Thus we have:

$$
\mathcal{T}_{A B}(k)=\int_{-\infty}^{\infty} \Phi_{A B}(k, t) \mathrm{d} t=g_{2}(k)\left\{1+\sum_{i=2}^{\infty} \frac{(-1)^{i}}{2^{i} i !} \frac{K_{2 i}(k)}{K_{2}^{i}(k)}\right\},
$$

where $g_{2}(k)$ denotes Gaussian contribution of TCFs in the additive approach form:

$$
g_{2}(k)=M_{0}(k) \sqrt{\frac{\pi}{2} \frac{M_{0}(k)}{K_{2}(k)}} .
$$

Now we are ready to consider all particular cases for $\{\hat{A}, \hat{B}\}=\{\hat{n}(\boldsymbol{k}), \hat{j}(\boldsymbol{k}), \hat{\varepsilon}(\boldsymbol{k})\}$.

To obtain the explicit expressions for cumulant expansion of TCFs and their zero order time moments, let us present the expressions for some SCFs in the limit $k^{*} \gg 1$ (the expressions for the other high-order SCFs, the reader could find in [7]): ${ }^{2}$

$$
F_{n n}(k, 0)=1 ; F_{n \varepsilon}(k, 0)=\frac{3}{2} T+\left\langle\phi_{i}\right\rangle ; F_{\varepsilon \varepsilon}(k, 0)=\frac{15}{4} T^{2}+3 T\left\langle\phi_{i}\right\rangle+\left\langle\phi_{i}^{2}\right\rangle ;
$$

\footnotetext{
${ }^{2}$ We use denotation $k^{*}=k \sigma_{\mathrm{LJ}}$ for a dimensionless wave vector where $\sigma_{\mathrm{LJ}}$ means the diameter of Lennard-Jones particle.
} 


$$
\begin{gathered}
\Phi_{j \varepsilon}(k, 0)=\mathrm{i} k T\left(\frac{5}{2} T+\left\langle\phi_{i}\right\rangle\right) ; \quad \Phi_{j j}(k, 0)=C_{j j}+3 T^{2} k^{2} \\
\Phi_{\dot{\varepsilon} \dot{\varepsilon}}(k, 0)=C_{\varepsilon \varepsilon}+\frac{T}{m}\left[\frac{35}{4} T^{2}+5 T\left\langle\phi_{i}\right\rangle+\left\langle\phi_{i}^{2}\right\rangle\right] k^{2} .
\end{gathered}
$$

In (13) we have introduced the following notations: $\phi_{i}=1 / 2 \sum_{j \neq i=1}^{N} U\left(\left|\boldsymbol{r}_{i}-\boldsymbol{r}_{j}\right|\right)$, $C_{j j}=1 / 3\left\langle\boldsymbol{F}_{i}^{2}\right\rangle \geqslant 0$, where dots mean the action of Liouville operator on the corresponding dynamic variables, $\boldsymbol{F}_{i}$ means the force acting on the i-th particle from the remaining molecules and the positive constant $C_{\varepsilon \varepsilon}$ is related to the derivative of the total energy of the i-th particle.

From the relations (13), it is seen that to calculate the corresponding SCF, one has to know the distribution function one order lower in comparison with the case of intermediate $k$. For instance, to calculate the static structure factor $S(k) \equiv \Phi_{n n}(k, 0)$ it is sufficient to know one-particle distribution function which is known to be equal to unity in the uniform case, the information about binary function allows one to evaluate $\Phi_{n \varepsilon}(k, 0)$ and to calculate $\Phi_{\varepsilon \varepsilon}(k, 0)$ it is necessary to know the triple distribution function.

Now let us turn our attention to the two last formulae in equation (13). The second terms in $\Phi_{j j}(k, 0)$ and $\Phi_{\dot{\varepsilon} \dot{\varepsilon}}(k, 0)$ are dealt with kinetic component of stress tensor and heat current respectively. They are of the leading order in the wave vector while the first terms describe the contribution of a potential component of the mentioned dynamic variables and form the so-called final state effects in neutron scattering experiments [8-10]. The other higher order SFCs involved in cumulants (9) have a similar form. In fact, they are series in $k$ where the leading term gives the main contribution in the corresponding TCFs.

Now we consider TCF "density-density". Taking use of the expression for SCFs one can write down the Gaussian contribution as follows:

$$
g_{2}^{n n}(k)=\frac{1}{k} \sqrt{\frac{\pi m}{2 T}} \sim\left(v_{\mathrm{T}} k\right)^{-1}
$$

where $v_{\mathrm{T}}=\sqrt{2 T / m}$ means the thermal velocity of the particle. The contributions of higher cumulants in (11) contain the only terms related to the final state effects which are found to be proportional to $k^{-3}$. The terms related to the kinetic components of the corresponding fluxes cancel each other for Maxwellian distribution that could be easily verified. When we have a quantum liquid with a distribution function in a momentum space which differs from Maxwellian form, the contributions from $K_{2 i}(k), i \geqslant 2$ are comparable with Gaussian part, and hence could be observable on the dynamic structure factor in high energy scattering experiments [8-10].

To calculate $\mathcal{T}_{n \varepsilon}(k)$, note that the main contribution in higher moments of $M_{2 s}^{n \varepsilon}(k)$ arising from kinetic parts of the fluxes could be calculated explicitly:

$$
M_{2 s}^{n \varepsilon}\left(k^{*} \gg 1\right)=\frac{\mathrm{i} k}{m} \frac{\left\langle(\mathrm{i} \hat{L})^{s} \hat{j}(\boldsymbol{k})(\mathrm{i} \hat{L})^{s-1} \hat{\varepsilon}(-\boldsymbol{k})\right\rangle}{\Phi_{n \varepsilon}(k, 0)}=\frac{k^{2 s} T^{s}}{m^{s}} \frac{s+1.5+x}{1.5+x}(2 s-1) ! !
$$


where we have introduced the notation $x=\left\langle\phi_{i}\right\rangle / T$ for a dimensionless mean potential. Now the expression for $\mathcal{T}_{n \varepsilon}(k)$ can be presented as follows:

$$
\mathcal{T}_{\text {ne }}(k)=g_{2}^{n \varepsilon}(k)\left\{1-\frac{3}{8} a^{2}-\frac{5}{8} a^{3}-\frac{105}{64} a^{4}+o\left(k^{-2}\right)\right\}, \quad a=(2.5+x)^{-1},
$$

where we have omitted cumulants higher than the 8-th while the Gaussian contribution equals

$$
g_{2}^{n \varepsilon}(k)=\frac{1}{k} \sqrt{\frac{\pi m T(1.5+x)^{3}}{2(2.5+x)}} .
$$

Note that the formal divergency of series (15) for slightly attractive potentials with $-2.5 \leqslant x \leqslant-1.5$ is only the result of an additive approach (10). When we operate with a more general form (7) for TCF, no divergency is observed but then we can evaluate a zero order time moment only numerically for each particular mean potential. Of course, one has to pay attention to the sign of the highest cumulant retained to obtain a convergent result (for instance, $K_{6}^{n \varepsilon}(k)$ is found to be negative for an attractive potential and this is true for slightly attractive potentials in case of $K_{6}^{\varepsilon \varepsilon}(k)$ as well).

In a similar way, having calculated higher moments for $\{A, B\}=\varepsilon$

$$
M_{2 s}^{\varepsilon \varepsilon}\left(k^{*} \gg 1\right)=\frac{k^{2 s} T^{s}}{m^{s}} \frac{(2 s-1) ! !}{3.75+3 x+y}\left\{y+(2 s+3) x+\frac{(2 s+3)(2 s+5)}{4}\right\},
$$

where $y=\left\langle\phi_{i}^{2}\right\rangle / T^{2}$ denotes the dimensionless second moment of potential, we can present zeroth time moment of TCS $\Phi_{\varepsilon \varepsilon}(k, t)$ in the explicit form:

$$
\begin{aligned}
& \mathcal{T}_{\varepsilon \varepsilon}(k)= \\
& =g_{2}^{\varepsilon \varepsilon}(k)\left\{1+\frac{3}{16}\left(4 y-8 x^{2}-28 x-35\right) b^{2}+\frac{5}{8}(x+2.5)\left(55+44 x+16 x^{2}-12 y\right) b^{3}\right. \\
& \quad-\frac{105}{512}\left[2225+x\left(3560+2544 x+896 x^{2}+128 x^{3}-544 y-128 x y\right)\right. \\
& \left.\quad+16 y(y-42.5)] b^{4}+o\left(k^{-2}\right)\right\}, \quad b=(8.75+5 x+y)^{-1},
\end{aligned}
$$

where we have for Gaussian contribution:

$$
g_{2}^{\varepsilon \varepsilon}(k)=\frac{T}{k} \sqrt{\frac{\pi m T(3.75+3 x+y)^{3}}{2(8.75+5 x+y)}} .
$$

The questions concerning the convergency rate for series (11) as well as the contribution of higher order cumulants could be answered separately for each particular interaction potential and they are not the subject of our interest. 


\section{Discussion}

In the framework of generalized collective modes approach [7] TCF $\Phi_{A B}(k, t)$ could be presented as a sum of the weighted exponent

$$
\Phi_{A B}(k, t)=\sum_{\alpha=1}^{M} G_{\alpha}^{A B}(k) \exp \left\{-z_{\alpha}(k) t\right\},
$$

where $z_{\alpha}(k)$ denote eigenvalues of a generalized dynamic matrix with elements consisting of SCFs and zero order time moments $\mathcal{T}_{A B}(k)$, amplitudes $G_{\alpha}^{A B}(k)$ are related to its eigenvectors and sum runs over all collective excitations $M$ depending on the number of dynamic variables for each particular model: $M=3$ for hydrodynamic level of description [6] when $\{\hat{A}, \hat{B}\}=\{\hat{n}(\boldsymbol{k}), \hat{j}(\boldsymbol{k}), \hat{\varepsilon}(\boldsymbol{k})\}, M=5$ for thermoviscous model when first derivatives of momentum and energy densities are included into the set (4)-(6) and so on.

Now taking derivatives of $\Phi_{A B}(k, t)$ in equation (20) with respect to time up to the s-th order and equating them with short time expansion of TCF in the limit $k^{*} \gg 1$, one can prove a collective excitation $z_{\alpha}(k)$ to be linear in a wave vector for all collective modes while the amplitudes $G_{\alpha}^{A B}(k)$ are found to approach their constant asymptotic values (note that the same result could be found by a direct investigation of generalized dynamic matrix spectrum in the high $k$ limit [7]). Moreover, there is a reciprocal correspondence between the number of dynamic variables considered and the order of frequency moment up to which the particular TCF is being correctly reproduced [11]). For instance, for a dynamic structure factor in the framework of 5 -variable thermoviscous model, one can obtain a correct expression up to the 4 -th frequency moment $[11,13]$.

The value of $k$ at which the excitation spectrum approaches its linear form is closely related to the incoherent scattering regime when final state effects could be neglected [8] and the dynamic structure factor takes its Gaussian form. The deviations of the other TCFs from Gaussian shape is caused by nonzero higher orders cumulants calculated in the previous Section. In nondegenerated quantum liquids due to the deviation of distribution function in momentum space from its Maxwellian form the Gaussian shape of $S_{\mathrm{sym}}(k, \omega)$ is distorted too [10]. The corresponding series are found to be well convergent and the contribution of higher cumulants could be neglected. We believe TCFs "energy-density" and "energy-energy" are well convergent for all realistic potentials but as we have already mentioned this could be the subject of a separate study.

\section{Acknowledgements}

Author would like to acknowledge granting the stipend of the President of Ukraine for young scientists. 


\title{
References
}

1. Hansen J.-P., McDonald I.R. Theory of Simple Liquids. London, Academic, 1986.

2. Price D.L. - In: The Physics of Liquid and Solid Helium. New York, Wiley Interscience, 1978.

3. Omelyan I.P. Generalized collective mode approach in the dielectric theory of dipolar systems. // Physica A, 1997, vol. 247, No. 1/4, p. 121-139.

4. Bryk T.M., Mryglod I.M., Kahl G. Generalized collective modes in a binary $\mathrm{He}_{0.65^{-}}$ $\mathrm{Ne}_{0.35}$ mixture. // Phys. Rev. E, 1997, vol 56, p. 2903-2915.

5. Lovesey S.W. Theory of Neutron Scattering from Condensed Matter. Oxford, Clarendon Press, 1984.

6. Boon J.-P., Yip S. Molecular Hydrodynamics. New York, McGraw-Hill, 1980.

7. Mryglod I.M, Omelyan I.P., Tokarchuk M.V. Generalized collective modes for the Lennard-Jones fluid. // Mol. Phys., 1995, vol. 84, No. 2, p. 235-259.

8. Glyde H.R. Excitations in Liquid and Solid Helium. Oxford, Clarendon Press, 1994.

9. Glyde H.R. Momentum distributions and final-state effects in neutron scattering. // Phys. Rev. B, 1994, vol. 50, p. 6726-6742.

10. Andersen K.H., Stigling W.G., Glyde H.R. Momentum distributions and final-state effects in liquid ${ }^{4}$ He. // Phys. Rev. B, 1997, vol. 56, p. 8978-8987.

11. Mryglod I.M. Generalized hydrodynamics of liquids. II. Time correlation functions in generalized collective modes approach. // Ukr. Phys. Journ., 1998, vol. 43, p. 252-256.

12. Cramér H. Mathematical Methods of Statistics. Princeton, Princeton University Press, 1979.

13. Ignatyuk V.V., Mryglod I.M., Tokarchuk M.V. On the theory of dynamic properties of semiquantum helium. // Low Temp. Phys., 1999, vol. 25, No. 5, p. 295-302.

14. Risken H. The Fokker-Planck Equation. Berlin, Springer-Verlag, 1984.

\section{Короткохвильова асимптотика часових кореляційних функцій}

\author{
В.В.Ігнатюк \\ Інститут фізики конденсованих систем НАН України, \\ 79011 Львів, вул. Свєнціцького, 1
}

Отримано 1 серпня 2000 p.

Короткочасова асимптотика часових кореляційних функцій (ЧКФ) простих рідин розглядається в рамках методу розкладу за малими часами. Вирази для нульових часових моментів з точністю до 8-го кумулянта представлені в наближенні адитивного підходу. Показано, що на відміну від динамічного структурного фактора, який має гаусову форму в короткохвильовій границі, в ЧКФ “густина-енергія" та "енергія-енергія" наявні негаусові поправки. Результати порівнюються 3 концепцією узагальнених колективних мод та даними для квантових рідин.

Ключові слова: часові кореляційні функції, короткохвильова границя, узагальнені колективні моди, кумулянтний розклад

PACS: $05.70 . \mathrm{Ln}, 51.10 .+y, 61.20 . L c, 61.20 . \mathrm{Ne}$ 